\title{
The Feasibility of Long Cycle Time Synchronization for Wireless Sensor Networks
}

\author{
Fuqiang Wang ${ }^{1,2, a}$, Xiaoming $\mathrm{Wu}^{3,1,2, \mathrm{~b}}$, Hong $\mathrm{Liu}^{1,2, \mathrm{c}}$, Yifan $\mathrm{Hu}^{1,2, \mathrm{~d}}$ and \\ Xiangzhi Liu ${ }^{1,2, \mathrm{e}}$ \\ ${ }^{1}$ Shandong Computer Science Center (National Supercomputer Center in Jinan), Shandong \\ Academy of Sciences, Jinan, China \\ ${ }^{2}$ Shandong Provincial Key Laboratory of Computer Networks, Jinan, China \\ ${ }^{3}$ College of Information Science and Engineering, Shandong University of Science and Technology, \\ Qingdao, China \\ awangfq@sdas.org, 'buxm@sdas.org, 'liuh@sdas.org, dhuyf@sdas.org, ${ }^{\mathrm{e}}$ liuxzh@sdas.org
}

Keywords: Wireless Sensor Networks, Time Synchronization, Low Energy Consumption, Precision

\begin{abstract}
Energy efficiency has received extensive attention in energy-constrained Wireless Sensor Networks (WSNs). As is known, radio communication accounts for major energy consumption of wireless device and the control of radio transmitter can make effective function with energy efficiency system. In this paper we test and analyse the relationship between precision and synchronization rate and we also give an initial model denote for the relationship among synchronous cycle, time error and energy consumption. The experiments and analysis in the paper show the feasibility of long cycle time synchronization for WSNs under certain limitation and the result will be helpful for the time synchronization method with low energy consumption.
\end{abstract}

\section{Introduction}

WSNs consist of large populations of wirelessly connected nodes, capable of computation, communication, and sensing. Although each sensor node is equipped with a hardware clock, these hardware clocks can usually not be used directly, as they suffer from severe drifts. No matter how well these hardware clocks will be calibrated at deployment, the clocks will ultimately exhibit a large skew. In order to get an accurate common time, nodes need to exchange messages from time to time, constantly adjusting their clock values.

Several time synchronization protocols have been developed to deal with the special requirements of WSNs' application. The typical time synchronization methods are TPSN (Timing-sync Protocol for Sensor Networks), RBS (Reference Broadcast Synchronization), FTSP (Flooding Time Synchronization Protocol), LTS (Lightweight Time Synchronization), Simple Time Synchronization, Tsync et al [1, 2]. The precision of some methods can reach microsecond-level. However, most of the protocols mentioned above utilize fixed synchronization rates that are acquired from experience value and may bring a large amount of messages interaction.

In this paper, we test and analyze the relationship between precision and synchronization rate and give an initial model denote for the relationship among synchronous cycle, time error and energy consumption to get the possibility of reducing energy consumption through maximizing the synchronization cycle under certain accuracy.

\section{Influences on Time-Synchronization}

Clocks in nodes, in general, based on crystal oscillators which provide a local time for each network node. The time in a node clock corresponds to a counter that gets incremented with crystal oscillators and is known as software clock. The software clock must be increased by the interrupt handler every time an interrupt occurs. Most hardware oscillators are not so precise because the frequency which 
makes time increase is never exactly right. For example if we use an oscillator with a frequency deviation of $0.001 \%$, the hardware clock would have a clock error of about one second per day ${ }^{[3]}$. The Node time expression is usually written as the equation $(3)^{[4]}$.

$$
T_{i}(t)=\beta_{i}+\lambda_{i} t+\gamma_{i} t^{2} \cdots
$$

Some protocols such as TPSN use constant model $\left(\alpha_{i}=0\right.$ and $\left.\gamma_{i}=0\right)$ that concern noting more than time offset between two clocks. Several protocols such as FTSP employ linear model $\left(\gamma_{i}=0\right)$ and estimation of $\alpha_{i}$ and $\beta_{i}$ is readily accomplished via linear regression (least squares). Such treatment method is optional if the error is Gaussian and the method can provide good estimator for the common error. However if the skew and offset are varying with time, the time model needs to be updated to a quadratic model. As $\alpha_{i}$ and $\beta_{i}$ change during the observation interval, the linear model would bring additional error. In this case, a quadratic model can be used to detect clock drift ${ }^{[5]}$.

Without concern the drift between two clocks, constant model should exchange messages from time to time, constantly adjusting their clock values. A quadratic model will lead to higher computational complexity and this is not suitable for resource constrained wireless sensor networks. Current state-of-the-art linear model is widely used for time synchronization in WSNs, and the design such as FTSP can optimize the clock skew to get good performance in WSNs.

Generally speaking, synchronization cycle and synchronization precision is a pair of contradictions exists: when synchronization cycle increases, synchronization accuracy is low, and vice versa. So it is very necessary to study the relationship between synchronization cycle and synchronization error.

\section{The Relationship between precision and synchronization Rate}

Since energy is an important factor for WSNs and the synchronization cycle will greatly effect energy consumption as depicted in [6], so it is essential to employ the cycle as large as possible to save energy. But this does not mean the bigger the better for the synchronization cycles, for the greater periods will bring the worse errors. We summarize the different synchronization period with different amount of synchronization points through off-line simulation, and the regression results are plotted as shown in Fig. 1. For the synchronization period equal and below 120 seconds, the average time error is always less than 1 time tick. However, when the synchronization periods grow more than 300 seconds, the fitting results would become worse with the increasing of fitting points. So our results can be used as a reference for selecting an optimal synchronization period.

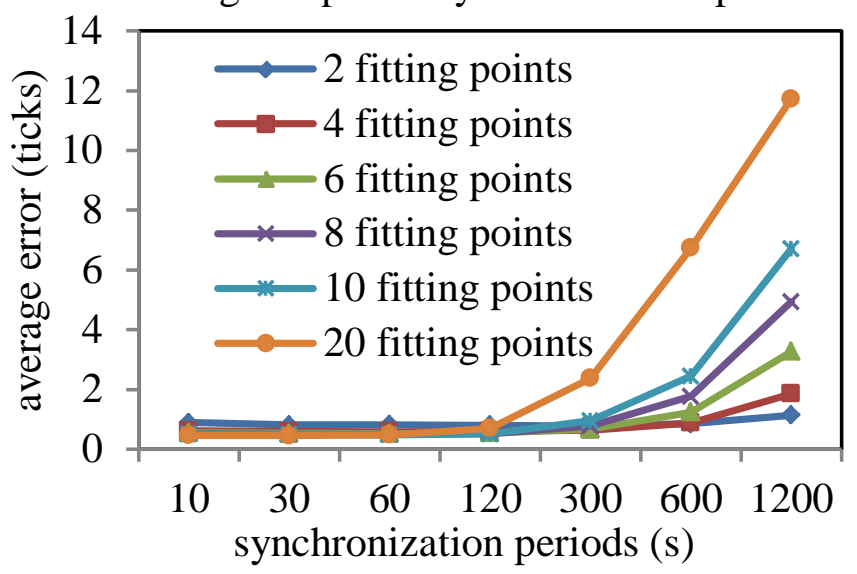

Fig 1. The results of the minimum distance square method in different synchronization periods

When the synchronization period is small, the regression results with more fitting points will get better performance but on the other hand (with larger period), a small number of fitting points will get better results. The statistical results of the maximum errors are the same case as depicted in Fig. 2. So there must be a balance for synchronization periods and the amount of fitting points that can get optional performance of time synchronization for WSNs. 


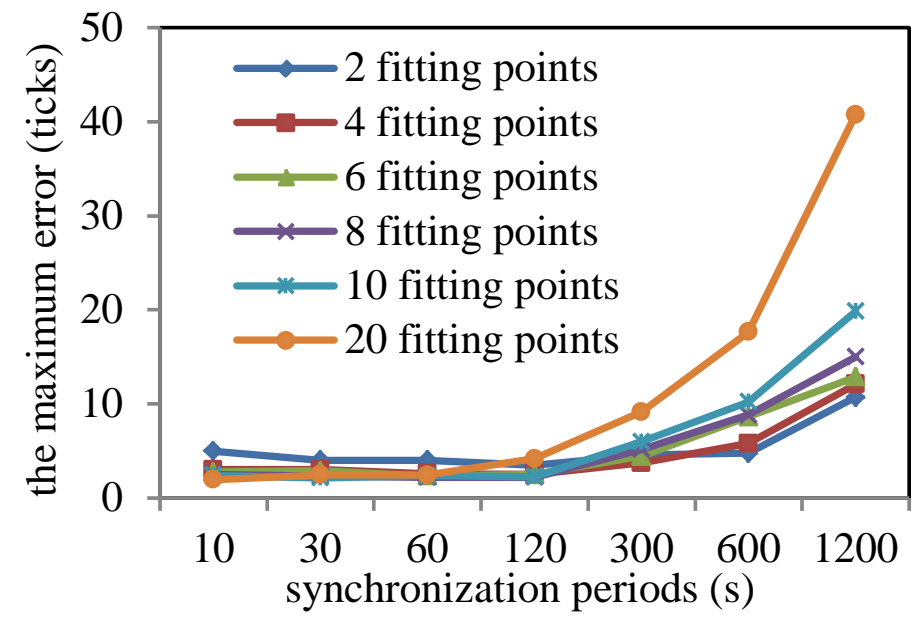

Fig 2. The maximum error distribution in different synchronization periods

We tested the time error for tens of nodes synchronized to the same reference node with different synchronization cycles and the result is shown in Fig.3. When the synchronization cycle is less than 360 seconds the time error grows slowly from 1 time tick to 3 ticks and the growing trend increases more sharply with the increasing of time cycle. So it is possible to maximize the synchronization cycle under certain demand for time error. But is it means larger time cycle is better for network performance? Of course not! The average additional current caused by time synchronization process is show in Fig. 4. The curve is got from the testing of MICAZ nodes synchronizing with a reference node under different cycles and from the profile the average additional current used for synchronization decreased sharply with the increasing of synchronization cycle. In the picture the trend of average current can be fitted to an exponential curve, which means when the time cycle is large the effect caused by the synchronization process will be extremely small.

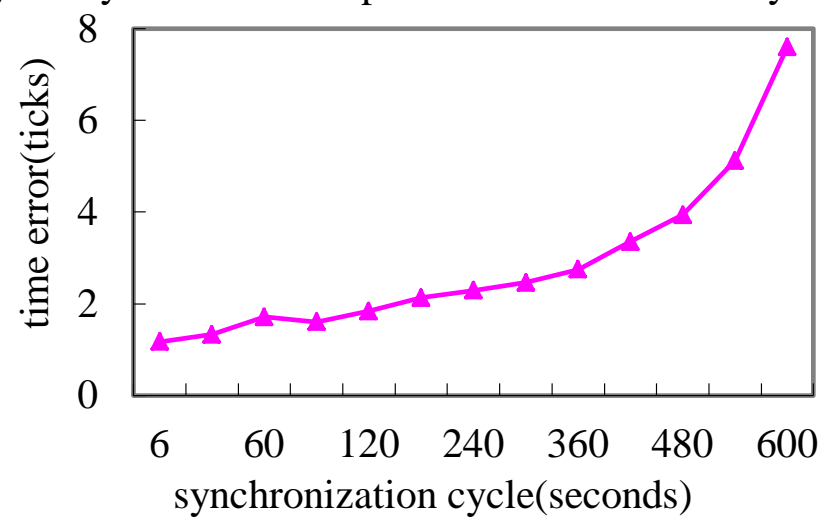

Fig 3. The average time error with different time cycles

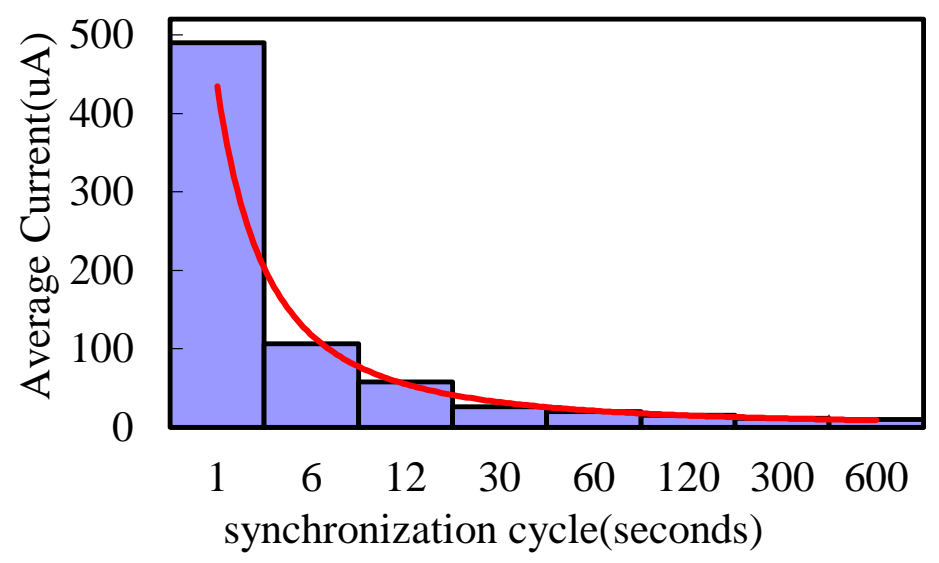

Fig 4. The average current with different time cycles 
Based on the above analysis, there exists a relationship between time error and synchronization cycle as shown in Fig 3 and the same situation between average current and synchronization cycle. These two relationships can be modeled in equation 4 . Here $y_{1}$ denotes as the time error of synchronization method with certain synchronization cycle $x$ and $y_{2}$ means average energy consumption (corresponding with the average current) changes with synchronization cycle $x$.

$$
\left\{\begin{array}{c}
y_{1}=a_{1} e^{n_{1} x}+b_{1} \\
y_{2}=a_{2} e^{-n_{2} x}+b_{2}
\end{array}\right.
$$

From above model, we can achieve a proper cycle when the demand time error $y_{1}<\Delta e$ and energy consumption constraint $y_{2}<\Delta E$. By this method we can get the scope of synchronous cycle as demanded and the model is effective for saving energy.

\section{Conclusions}

This paper makes a simple analysis of the relationship among synchronous cycle, time error and energy consumption and an initial model of the relationship is given in the paper. The experiments and analysis in the paper show the feasibility of long cycle time synchronization for WSNs under certain limitation and the result will be helpful for the time synchronization method with low energy consumption. Further research may consider additional energy consumption caused by unreliable links that contribute to the synchronization overhead and accuracy

\section{Acknowledgements}

This work was financially supported by the Shandong Academy of Sciences Pilot Project for Science and Technology, and basic research fund of Shandong Academy of Sciences (No. 2015[25]), National Natural Science Foundation of China (No. 61501282, 61401257)

\section{References}

[1] Sarvghadi M.A. and Tat-Chee Wan, Overview of time synchronization protocols in wireless sensor networks. the 2nd International Conference on Electronic Design (ICED), 2014, pp. 204-209.

[2] Wang F, Yu C, Wu X, et al, Dual time synchronisation method for wireless sensor networks. Electronics Letters, 51(2), 2015, pp. 179-181.

[3] Rhee, I. K., Lee, J., Kim, J., Serpedin, E., \& Wu, Y. C, Clock Synchronization in Wireless Sensor Networks: An overview. Sensors, 9(1), 2009, pp. 56-85.

[4] Wang F, Wu X, Pang Y, et al, A time synchronization method of Wireless Sensor Networks based on the simulated annealing algorithm, The 26th Chinese Control and Decision Conference (2014 CCDC), 2014, pp. 870-875.

[5] Huang, G., Zomaya, A. Y., Delicato, F. C., \& Pires, P. F, Long term and large scale time synchronization in wireless sensor networks. Computer Communications, 37, 2014, pp. 77-91.

[6] Ganeriwal, S., Tsigkogiannis, I., Shim, H., Tsiatsis, V., Srivastava, M. B., \& Ganesan, D, Estimating clock uncertainty for efficient duty-cycling in sensor networks. IEEE/ACM Transactions on Networking (TON), 17(3), 2009, pp. 843-856. 